\title{
ECuADOR - Easy Curation of Angiosperm Duplicated Organellar Regions, a tool for cleaning and curating plastomes assembled from next generation sequencing pipelines
}

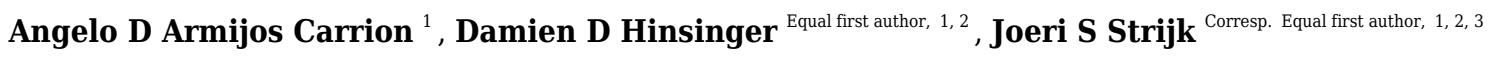 \\ 1 Biodiversity Genomics Team, Plant Ecophysiology \& Evolution Group, Guangxi Key Laboratory of Forest Ecology and Conservation, College of Forestry, \\ Guangxi University, Nanning, Guangxi, PR China \\ 2 Alliance for Conservation Tree Genomics, Pha Tad Ke Botanical Garden, Luang Prabang, Laos \\ ${ }^{3}$ State Key Laboratory for Conservation and Utilization of Subtropical Agro-bioresources, Guangxi University, Nanning, Guangxi, PR China \\ Corresponding Author: Joeri S Strijk \\ Email address: jsstrijk@hotmail.com
}

\section{Background:}

With the rapid increase in availability of genomic resources offered by Next-Generation Sequencing (NGS) and the availability of free online genomic databases, efficient and standardised metadata curation approaches have become increasingly critical for the post-processing stages of biological data. Especially in organelle-based studies using circular chloroplast genome datasets, the assembly of the main structural regions in random order and orientation represents a major limitation in our ability to easily generate "ready-to-align" datasets for phylogenetic reconstruction, at both small and large taxonomic scales. In addition, current practices discard the most variable regions of the genomes to facilitate the alignment of the remaining coding regions. Nevertheless, no software is currently available to perform curation to such a degree, through simple detection, organization and positioning of the main plastome regions, making it a time-consuming and error-prone process. Here we introduce a fast and user friendly software ECUADOR, a Perl script specifically designed to automate the detection and reorganization of newly assembled plastomes obtained from any source available (NGS, sanger sequencing or assembler output).

\section{Methods:}

ECUADOR uses a sliding-window approach to detect long repeated sequences in draft sequences, which then identifies the inverted repeat regions (IRs), even in case of artifactual breaks or sequencing errors, and automates the rearrangement of the sequence to the widely used LSC-Irb-SSC-IRa order. This facilitates rapid post-editing steps such as creation of genome alignments, detection of variable regions, SNP detection and phylogenomic analyses.

\section{Results:}

ECUADOR was successfully tested on plant families throughout the angiosperm phylogeny by curating 161 chloroplast datasets. ECUADOR first identified and reordered the central regions (LSC-Irb-SSC-IRa) for each dataset and then produced a new annotation for the chloroplast sequences. The process took less than 20 minutes with a maximum memory requirement of $150 \mathrm{MB}$, and an accuracy of over $99 \%$. 


\section{Conclusions:}

ECUADOR is the sole de novo one-step recognition and re-ordination tool that provides facilitation in the post-processing analysis of the extra nuclear genomes from NGS data. The program is available at https://github.com/BiodivGenomic/ECuADOR/. 
1

2 ECuADOR - Easy Curation of Angiosperm Duplicated Organellar Regions, a tool for cleaning

3 and curating plastomes assembled from next generation sequencing pipelines

4

5 Angelo Damian Armijos Carrion ${ }^{1,+}$; Damien Daniel Hinsinger ${ }^{1,3,+}$; Joeri Sergej Strijk $k^{1,2,3,+, *}$

$7{ }^{1}$ Biodiversity Genomics Team, Plant Ecophysiology \& Evolution Group, Guangxi Key

8 Laboratory of Forest Ecology and Conservation, College of Forestry, Guangxi University,

9 Daxuedonglu 100, Nanning, Guangxi, 530005, PR China

$10^{2}$ State Key Laboratory for Conservation and Utilization of Subtropical Agro-bioresources,

11 Guangxi University, Nanning, Guangxi 530005, PR China

$12{ }^{3}$ Alliance for Conservation Tree Genomics, Pha Tad Ke Botanical Garden, PO Box 959, 06000

13 Luang Prabang, Laos

14

$15 \dagger$ Authors contributed equally

16

17 Corresponding Author:

18 Joeri Sergej Strijk $k^{1,2,3}$

19 Guangxi University, 100 DaXueDongLu, Nanning, Guangxi Province, 530005, PR China

20 Email address: jsstrijk@hotmail.com

21

22

23

24

25 
26 Abstract

27 Background:

28 With the rapid increase in availability of genomic resources offered by Next-Generation

29 Sequencing (NGS) and the availability of free online genomic databases, efficient and 30 standardised metadata curation approaches have become increasingly critical for the post31 processing stages of biological data. Especially in organelle-based studies using circular 32 chloroplast genome datasets, the assembly of the main structural regions in random order and 33 orientation represents a major limitation in our ability to easily generate "ready-to-align” datasets 34 for phylogenetic reconstruction, at both small and large taxonomic scales. In addition, current 35 practices discard the most variable regions of the genomes to facilitate the alignment of the 36 remaining coding regions. Nevertheless, no software is currently available to perform curation to 37 such a degree, through simple detection, organization and positioning of the main plastome 38 regions, making it a time-consuming and error-prone process. Here we introduce a fast and user 39 friendly software ECuADOR, a Perl script specifically designed to automate the detection and 40 reorganization of newly assembled plastomes obtained from any source available (NGS, sanger 41 sequencing or assembler output).

\section{Methods:}

$43 E C u A D O R$ uses a sliding-window approach to detect long repeated sequences in draft sequences, 44 which then identifies the inverted repeat regions (IRs), even in case of artifactual breaks or 45 sequencing errors, and automates the rearrangement of the sequence to the widely used LSC-IRb46 SSC-IRa order. This facilitates rapid post-editing steps such as creation of genome alignments, 47 detection of variable regions, SNP detection and phylogenomic analyses.

48 Results:

$49 E C U A D O R$ was successfully tested on plant families throughout the angiosperm phylogeny by 50 curating 161 chloroplast datasets. ECUADOR first identified and reordered the central regions 51 (LSC-IRb-SSC-IRa) for each dataset and then produced a new annotation for the chloroplast 52 sequences. The process took less than 20 minutes with a maximum memory requirement of 150 $53 \mathrm{MB}$, and an accuracy of over $99 \%$.

\section{Conclusions:}

$55 E C U A D O R$ is the sole de novo one-step recognition and reordination tool that provides facilitation

56 in the post-processing analysis of the extra nuclear genomes from NGS data. The program is 57 available at https://github.com/BiodivGenomic/ECuADOR/. 
Introduction

59 Chloroplast DNA (cpDNA) has been used extensively in plant phylogenetic studies as it is

60 maternally inherited in most angiosperms, has a low mutation rate and provides variable and

61 informative regions over broad timescales [1, 2]. Moreover, cpDNA provides abundant DNA

62 polymorphisms at inter- and intraspecific levels, providing molecular phylogenies with high

63 resolution at different taxonomic scales [3].

64 The emergence of next generation sequencing (NGS) technologies, allowing for complete

65 organellar DNA sequencing, has promoted the use of plastome (i.e. complete chloroplast DNA

66 sequences) data as a major tool in phylogenomic and evolutionary analyses [4, 5], as well as an

67 extended DNA-barcode [6]. As a result, massive amounts of genome-scale data have been

68 generated at a relatively low cost, with ever faster turnaround times [5]. The availability of this

69 expanding data volume has allowed for the exploration of chloroplast organization and

70 chloroplast features throughout the angiosperm tree at the molecular level [7], and the

71 development of novel approaches for phylogenetic studies [8]. To date, for angiosperms alone,

72 there are over 4500 sequenced plastomes available in NCBI GenBank through the INSDC

73 database (as of June 2019) and this number has seen an exponential increase in recent years.

74 A consequence of the rapid development of NGS techniques is the emerging need to handle and

75 curate increasingly large organellar genome datasets. For example, a recent study [9], used nearly

76 3,000 plastomes to reconstruct angiosperm phylogeny and evolution. Because mitochondrial

77 genomes and plastomes are usually circular, but are represented as linear text sequences in

78 bioinformatic pipelines and online databases, any base position can represent the start of the

79 string sequence (see Figure 1). Plastome sequences are typically characterized by two inverted-

80 repeated regions (hereafter called IRs) separated by a long (LSC) and a short (SSC) single copy

81 region $[10,11]$. Due to the replication mode of organellar DNA, each part can be found in both

82 directions (i.e in 5'->3' orientation, or in the reverse complement orientation) in the cell [10].

83 This organization implies that orthologous regions need to be assessed for each of these parts

84 (Figure 1). By general consensus, and some computational constraints, most of the assembly

85 methods start the reconstructed sequence at the first base of the large single-copy region (LSC).

86 However, depending on both the quality and quantity of the NGS sequences (i.e. the "raw

87 reads”), as well as the complexity of the plastome to reconstruct, a sequence may start randomly

88 along the circular sequence (Figure 1b), occasionally making the identification of homologous

89 regions among sequences laborious and time-consuming, especially for large datasets (100-1000s

90 of sequences). 
91 Despite there being some consensus in the community on the starting position of plastome 92 sequences, a quick assessment of sequences in GenBank shows extensive disparity in the 93 organization and order of the different regions. To circumvent this, most of the plastomic studies 94 [30] (including the above-mentioned 3,000 plastomes study [9]) only consider the coding regions 95 of the reconstructed organelles, to remove small inversions and changes in gene order. However, 96 such rearrangements are less likely to happen at small taxonomic scales (e.g. at intra-generic 97 level, or in species complexes). Moreover, such an approach results in the deletion of the non98 coding regions, that often contain more phylogenetic signal [12], especially at lower taxonomic 99 level or in recalcitrant taxa, in which genomic approaches are usually required to fully resolve 100 relationships $[13,14]$. In addition, using the entire plastome sequence allows for detection of 101 evolutionary events, such as insertion-deletions or inversions, and facilitate the identification of 102 sequences from non-organelles origins (nuclear plastid DNA - NUPTs ; nuclear mitochondrial 103 DNA - NUMTs).

104 Accurate and complete molecular sequence data are essential in phylogenomic reconstruction. 105 Therefore, accurate homology detection among sequences is a vital step. Consequently, 106 improvement in the functional annotation of organisms will become an extremely important step 107 to delineate evolutionary processes.

108 Usually, three distinct steps can be identified in phylogenomic studies, 1) the reconstruction of 109 organelles in the different species; 2) the assessment of orthology and alignment of the plastome 110 sequences (i.e. the dataset assembly); and 3) the actual phylogenetic reconstruction [15]. Current 111 software focuses on organelle reconstruction [e.g 16, 17], multiple sequence alignment (MSA) 112 algorithms [e.g 18] and phylogenetic approaches [e.g. 19]. Software currently available can aid in 113 assembly and analyses of plastid and mitochondrial genomes. However, orthology assessment 114 and curation of the assembled plastomes usually remains a manual task [but see [29] for a 115 combined assembly and orientation script]. Indeed, there is currently no open-source software 116 that reconstructs and assesses plastome orthology for large datasets (numbering thousands of 117 plastomes) in a fast and accurate way from assembled draft sequences. While orthology 118 assessment can be easily done for datasets based on individually extracted coding regions, the 119 specific structure of the plastome (LSC,IRb,SSC,IRa) complicates the proper alignment of 120 orthologous regions. Indeed, any differences in organization/orientation of these parts in the 121 reconstructed draft plastomes, can result in improper assessment of orthologous positions and 122 thus increase the risk of discarding potentially useful data at the end of alignments. This process 123 is either done manually (representing 3-5 minutes per chloroplast for a trained bioinformatician, 
124 using commercially available software, e.g Geneious [21]), or skipped by extracting the coding

125 regions (thus discarding the most variable and useful regions of the plastome - see above). This

126 represents an emergent bottleneck in dataset assembly and downstream analyses. Therefore, the 127 development of flexible and user-friendly tools that remove these labor-intensive and time-

128 consuming components from genomic workflows has become a latent priority for the plant 129 genomics community.

130 We designed ECUADOR to facilitate both the rapid processing of organelle genomic data as well

131 as providing output requirements for downstream analyses. ECUADOR is a rapid, platform-

132 independent and user-friendly algorithm built in Perl, that automates detection and reorganization

133 of sequence features in newly assembled plastomes. As ECUADOR uses only the reconstructed 134 plastomes, it is also independent of the sequencing technology used to generate the data, and can 135 thus be used with assemblies derived from short reads (e.g. Illumina), long reads (e.g. PacBio, 136 Nanopore) or those acquired using other sequencing technologies. Data are generated as moving 137 singular reciprocally-compared fragments, tracking the number of nucleotide changes for a 138 window of a user-defined length, slide along the sequence.

140 The algorithm is executed with default settings for the sliding window option (but with the option 141 to adjust these manually) and adjustments can be made to input parameters according to the 142 desired output format file (see ECuADOR command line below). These options can be 143 customized via a command-line, making it user-friendly and easily accessible.

\section{Materials \& Methods}

\section{Analysis pipeline}

$149 E C u A D O R$ is written in Perl (tested with Perl 5.18) and uses the following modules: Bio::SeqIO, 150 IO::String, Set::IntSpan, IO::File, Bio::AlignIO, Bio::Factory::EMBOSS, File::Temp $151 \mathrm{qw} / \mathrm{tmpnam} /$ and Cwd. Input for ECuADOR is a draft plastome sequence (GenBank or fasta), the 152 length of the sliding window, format of input file (GenBank or fasta), and output format file 153 (fasta or GFF3). ECUADOR reads and analyzes single or multiple plastomes stored in a 154 designated folder, containing one sequence per file. 
156 ECUADOR is based on a user-defined sliding window and dynamic suffix array approach. The

157 algorithm partitions the sequence into fragment intervals, of sizes defined by the "window size"

158 option. This window slides along the plastome in both 5' ->3' and 3' ->5' direction (as a reverse-

159 complement sequence). Then, a positioning array index is generated from the similarity between

160 the generated fragments. This new array index stores a sequence of the four main regions

161 according to the exact location where each repeated extreme was found. In later stages, the

162 extremes are used to recover the remaining IR length between both of them.

163 However, when the quality of the used sequencing reads is poor, mis-assemblies are likely to be

164 introduced in the sequence during organellar reconstruction (e.g. gapped palindromes), for

165 example in one of the repeats. In such cases, the sliding-window approach could retrieve different

166 sequence lengths for each repeat region. Because the main goal of the sliding-window approach

167 is to capture the two identical-sized repeat regions (IRa and IRb), a non-equality in any repeat

168 sequence length could prevent the recovery of its total length. This would result in an incorrect

169 positioning for all the main regions (LSC-IRb-SSC-IRa) and prohibit the rapid assembly of

170 correct alignments downstream. ECUADOR addresses this drawback by only using the extremes

171 of each inverted repeat to estimate the repeat size, i.e. once the position of each extreme fragment

172 is known in both repeats, ECuADOR will take all the remaining positions between both

173 previously positioned extremes to recover the entire repeated sequence. This offers a flexible

174 (allowing small discrepancy between IRs - value defined as a user-customizable setting) yet

175 conservative approach (the borders of IRs have to perfectly match each other).

$176 E C U A D O R$ allows the user to define the size of the window (in bps) used for the sliding-window

177 step. The optimal length of the sliding window (-w parameter) depends on the quality of the

178 reconstructed sequence. If the sequence quality is low, it is advised to use a smaller sliding

179 window size to adjust for the higher likelihood of finding gapped palindromes throughout the

180 inverted repeat. These would not normally be detected using a larger sliding window size, leading

181 to a loss of information in the length of the repeats. By adjusting the sliding window size, the user

182 can balance the sensitivity of the IR detection. A larger window size allows for reducing the

183 influence of misassembled reads and thus false positives - at the cost of a lower resolution (-

184 sensitivity, + specificity). A smaller window size provides increased resolution but may also

185 increase the number of false positives if the data is noisy (+ sensitivity, - specificity). The quality

186 of the input plastome sequence (e.g. as a consequence of a low quality base call during

187 sequencing or mis-assemblies) is an important factor to take into account before setting a value

188 for -w (i.e. the window size parameter). 
189 Finally, ECUADOR is executed using the following command-line perl command: ECuADOR.pl 190 [-h] -i, <folder containing the plastomes>, -w, <sliding window length> (1000 bp option by 191 default), -f, <input file format (option not by default, GenBank or fasta)>, -out < output prefix>, -192 ext, <output format> (either fasta or GFF3), --save_regions, <save chloroplast regions (LSC,

193 SSC, IRs separately, or in combination)>, --orient <TRUE> (reorientation of each plastome 194 regions, providing the user with the cpDNA regions ready to use for MSA), finally the option -195 noIRs $<3$ or $4>$ (to generate files including either only the concatenated regions LSC-IRb-SSC or 196 the entire one LSC-IRb-SSC-IRa)

198 The program is divided into seven main steps (Figure 2):

1. Argument check and analysis of the input file, setting format files and sliding window size (if no slide window size is provided, the default option will be set to $1000 \mathrm{bp}$.

2. ECUADOR generates a reverse complement sequence of the input fasta sequence (i.e. reconstructed draft plastome).

3. A suffix array index (permutation of index numbers giving the starting positions of suffixes of a string in alphabetical order) is built, comparing the suffix of fragment sequences with each and every suffix of the reverse complement fragments (both generated through slide windows). This new array index will be used to detect the exact location of the corresponding extremes to each IR. In the usual case (gap-free or identical palindromes), a total of 2 arrangements of only one sequential element will be located in the suffix arrays, thus covering the exact position of each repeat. In unusual cases (gapped palindromes) two arrangements of multi non-sequential elements will be located in the suffix arrays. In such cases these extra fragments will prevent the recovery of the repeated regions.

4. If three or more un-sequential elements are detected in any of the two main arrangements as a result of gapped palindromes, ECUADOR takes the extra smallest dissimilar sequential elements and it will create a new fragment from them. This new fragment will contain the start position of the multi non-sequential elements located in the first inverted repeat with the end position of the multi non-sequential elements located in the second inverted repeat. Then, this new fragment will be concatenated with the ending homologous fragment. 
221

222

223

224

225

226

227

228

229

230

231

232

233

234

235

236

237

238

239

240

241

242

243

\section{Taxon selection and dataset construction}

245 To test and assess performance of ECuADOR, two main datasets and a reference case were

246

247

248

249

250

251

252

253

5. Once the inverted repeat regions are located, the algorithm maps the remaining positions (LSC, and SSC respectively) through a sequence sweep from start to end.

6. When all the four main regions are found through mapping-by similarity-analysis (LSC, IRa, SSC, and IRb respectively), ECuADOR reorganizes the structure of the entire sequence in the same order for all the analyzed plastomes.

7. ECuADOR generates 5 output files. The first one is a summary of the main annotation features of the regions, including the length of the IRs. The second one is the reordered sequences (i.e. LSC-IRb-SSC-IRa) in either fasta or GFF3 format, depending on user options (for GenBank files, it will provide a complete reorientation and extraction for all its established annotations in a new GFF3 output file). If a sequence cannot be reliably curated, ECUADOR generates a third file containing a problem description for that particular sequence. If the option "all-orient TRUE” was selected, an additional step is performed, that will homogeneize the direction of the sequences for each plastome fragment before generating the final results files (for this purpose, we integrated in the ECUADOR code a modified version of the script seqOrient.pl, available at: http://raven.wrrb.uaf.edu/ ntakebay/teaching/programming/perl-scripts/seqOrient.pl). Finally, we included an additional option "-noIRs" (number of inverted repeats) which allows the user to get either one or two IRs. The default value is 1 (thus outputting the LSC-IRb-SSC), while “-noIRs 2” will output the complete plastome sequence (LSC-IRbSSC-IRa, useful e.g. before annotation for GenBank submission). The output (fasta file) can be immediately used in software for downstream phylogenetic and genomic analysis (e.g. Geneious, CLC workbench or MAFFT, PHYML and BEAST). generated. The first (dataset1: data control) was used to re-evaluate sequences corresponding to 161 published plastomes from a selection of 51 major angiosperm groups [3]. Fasta sequences of the plastomes were downloaded from GenBank, and analyzed using ECUADOR with default parameters and a sliding window adjusted to 1500 bp. We reviewed the accuracy of IR identification by evaluating the similarity and position of each retrieved region compared to their original annotation, using Geneious R9 v.9.0.5 [21], with the "Find repeats” function [1,500bp as minimum repeat length; no allowed mismatch between repeats]. To further evaluate the quality of the obtained locations (LSC, SSC, IRs), we built a phylogenetic tree based on the 161 reanalyzed 
254 plastomes, and compared this with the previously published topology [3]. The sequences were

255 aligned using MAFFT v.7 [22] under the FFT-NS-1 option, and PhyML v3.3.20 [23] was used to

256 build a maximum-likelihood (ML) tree with the GTR DNA substitution model and the fast

257 likelihood based method (aLRT SH-like).

258 Secondly, to assess the robustness of our algorithm, we simulated low quality/noisy plastomes by

259 introducing random substitutions with different percentages of variation in the plastome of 260 Arabidopsis thaliana (dataset2: data testing). Eleven levels of variation were chosen, ranging 261 from $0.01 \%$ to $5.31 \%$, and 1,000 simulations were generated for each variation level, 262 respectively, using an in-house script (available

263 https:/github.com/BiodivGenomic/ECuADOR/). These 'low quality' plastomes were then 264 evaluated through ECUADOR with default parameters.

265 Finally, in order to evaluate the applicability of our algorithm to any kind of chloroplast data (not 266 only for families previously analyzed) and taking advantage of its fast detection and extraction 267 speed, a dataset corresponding to 4541 angiosperm chloroplasts was downloaded from GenBank 268 (database INSDC accessed on 2019/06/20 - dataset3: mass data evaluation) and analyzed using 269 default options in the same format. This further served as an additional survey to detect cases 270 with missing or poorly uploaded regions currently available in GenBank. The goal of this 271 analysis was to evaluate the percentage of negative cases (i.e. without any IR identified), using 272 the largest number of chloroplasts available for angiosperms to date and to determine the 273 underlying causes of these events.

275 Results

276 Prediction of performance analysis (Dataset1: data control)

277 To evaluate ECUADOR, we compiled 161 plastome sequences from a total of 51 major 278 angiosperm groups, as previously used in [3]. ECuADOR ran fluently for each plastome, with 279 regions identified in almost all sequences (Supplementary Table S1). It provided basic 280 information regarding the location and inverted repeat lengths, reordering of the main plastome 281 regions (LSC-IRb-SSC-IRa) as well as the repositioning for all the protein coding genes (CDS) in 282 gff3 format file for all plastomes analyzed. Furthermore, it substantially eases the post-processing 283 analyses of plastomes reconstructed from NGS data. Results obtained with ECuADOR and 284 Geneious were very similar, validating the performance and accuracy of our approach 285 (Supplementary Table S1). Retrieved annotations were identical for 150 sequences out of 161. 286 This number increased to 160 after manually setting the first position of the LSC as the start of 
287 the plastome (contrary to $E C u A D O R$, in which this is done automatically). IR annotations were 288 not retrieved accurately for one draft plastome sequence (GU592211), due to very poor sequence 289 quality.

290 ECUADOR took 15 min to analyze this dataset using a MacBook Pro, 2.2 GHz Core 2 Duo, 16Gb

291 RAM. When compared to manual individual treatment (e.g. average of 3-5 min handling per 292 plastome), this would have taken between 8 and 13 hours.

293 The topology of the obtained phylogenetic tree (Figure 3) was similar for 49 families compared 294 to previously identified relationships [3]. An inconsistency was found in the placement of 295 Ranunculaceae (Ranunculus macranthus), which grouped together with Piperaceae, 296 Dioscoreaceae and Chloranthaceae.

\section{Performance of the introduced variation simulation (Dataset2: data testing)}

299 The introduction of mismatches between both repeats is based on the loss of information, thus 300 reducing the identity of the IRs and altering the final reorganization of the plastome. This 301 analysis allowed us to understand how the introduced error for the different simulation sets 302 affects the recovery of the original positions of the inverted repeats and therefore the ability of 303 the algorithm to retrieve the reordinated sequence completely. Thus, for each mismatch level, we 304 scored and investigated cases where ECUADOR failed to retrieve the original IRs locations (due 305 to excessive variation in the base pair numbers within the inverted repeats), using as a model the 306 Arabidopsis thaliana chloroplast genome (Figure 4).

$307 E C U A D O R$ was able to recover and reorder the main regions of the plastomes (LSC-IRb-SSC308 IRa) for each altered dataset. As expected, the mismatching percentage affecting the true 309 annotation increased as the alteration for each data set increased. ECUADOR showed an accuracy 310 above 90\% with 22 or fewer alterations (Supplementary Table S2). Such a high level of 311 mismatch between the two IRs, likely represents major misassembled positions and we 312 recommend such low-quality draft plastomes should first be carefully checked to assess the origin 313 of such mismatches. Moreover, the user can easily modify the stringency of the detection process

314 by specifying the sliding window fragment size to improve search precision. This should 315 however only be done for noisy datasets which are known to contain high levels of mismatching 316 or highly similar fragments throughout the plastome.

\section{Reference data set (Dataset3: mass data evaluation)}


319 To evaluate the potential and flexibility of ECUADOR, we analyzed a total of 4541 angiosperm

320 plastomes using default parameters with fasta format as input. The main regions were

321 successfully detected in 4446 sequences (97.90 \%, Supplementary file S1) whereas identification

322 and organization of the plastome structure failed in 95 sequences (2.09\%, Supplementary file 323 S2).

324 To further assess the causes of these failed instances, the 95 sequences were evaluated in 325 Geneious [21], and we found manual curation was needed to identify the main regions in these 326 plastomes. Indeed, these plastomes had missing regions or poorly formatted annotations in 327 GenBank, or were of extremely poor quality. Finally, several plastomes were characterized by an 328 absence of inverted repeats (e.g. gymnosperm plastomes and several species in parasitic plants $329[24,25])$.

$330 E C u A D O R$ requires a correct match between both repeats to be able to recover the newly 331 generated regions (LSC-IRb-SSC-IRa) and score the analysis as successful. The script will not 332 identify inverted repeat regions in sequences of extremely poor quality. Nevertheless, it has 333 several detection error and correction mechanisms - such as a wide detection of more than one 334 inverted repeat region and recognition of ambiguous characters in the plastome.

336 Discussion

$337 E C U A D O R$ is a novel algorithm designed for the identification, reorganization and reordering of 338 homologous regions (LSC, SSC and IRs) on large-scale plant datasets using the specific location 339 of the inverted repeats in any point of the circular genome as a starting point. Other software 340 aimed at helping with circular genomes exist, but none handles the post-assembly curation in a 341 standardized manner. For example, Circlator [26] was designed to use with long sequencing 342 reads (e.g. PacBio data) in bacterial chromosomes and plasmids and the plastid and mitochondrial 343 genomes of eukaryotes to reconstruct circular genomes. However, Circlator is an assembler and 344 thus works with the filtered, long sequencing reads (FASTQ files) to generate a circular genome. 345 In that way, it is not different from the many assemblers or scripts specifically designed to 346 generate organelles genomes (e.g. ORG.asm, NovoPlasty, GetOrganelle) that can be used before 347 ECUADOR. As most phylogenomic studies combine both new sequences and plastomes mined 348 from GenBank, original raw data can be missing for a significant part of the sampling. Although 349 long sequencing reads are quickly emerging as a powerful tool in genomics, the vast majority of 350 generated data available are short reads (<300bp) from Illumina platforms, prohibiting the use of 
351 a long-read-specific solution. As Circlator could be involved in the assembly process,

352 GenomeRing [27] is a visualization tool that allows an overview of several plastomes in the same

353 coordinate system. In that sense, it could be considered as a good complement to ECuADOR, to

354 visualize the results after alignment. But given that an alignment is a requirement for

355 GenomeRing and the program does not generate any files for immediate downstream analyses,

356 we cannot consider GenomeRing as an alternative for ECuADOR. The closest algorithm to

357 ECUADOR would be MARS [28], as it can homogeneize the starting point of a set of sequences

358 from a circular genome, a function ECUADOR also performs as a side-effect of the reorganization

359 of the plastome. However, ECuADOR is able to not only move the starting point, but also to keep

360 order and orientation of the main regions of the plastome. In addition, MARS outputs a FASTA

361 file, while ECUADOR can keep track of all annotations previously included in the input files.

362 Fast-Plast [29], despite being a “all-in-one” pipeline including assembly and ordering of the

363 plastome, is not able to handle GenBank sequences, limiting its use to newly generated draft

364 plastomes. In addition, its structure implies to work with each plastome separately, while

$365 E C u A D O R$ is able to homogeneize the output order and direction for a complete set of sequences,

366 making downstream analyses easier.

367 A possible explanation for the incongruence observed in dataset1 could be that Ruhfel et al. [3]

368 used only protein coding data of 78 genes from 360 taxa to build their phylogenetic tree. Gene

369 conflicts in plastome-based phylogenies have recently been highlighted as a major cause of

370 incongruence among studies [31, 32], and differences in methodology between our study and the

371 study by Ruhfel et al. [3] could explain the observed incongruence. The analyses performed here

372 were based on complete plastome data and include non-coding, fast evolving regions. Despite

373 that these regions have been proven useful at smaller taxonomic scales, they are expected to

374 saturate at very large scales, resulting in nonspecific phylogenetic signals in deeper parts of the

375 tree and causing incongruence with signals inherent in coding-regions. It is beyond the scope of

376 our study to provide a detailed analysis of the problems inherent in the reconstruction of the

377 phylogeny of angiosperms but worthy to note that the level of saturation effects in the plastome

378 dataset seems low, with only one incongruence compared to the analysis using coding-regions

379 only.

380 The introduction of ECUADOR has provided a major step forward in our ability to quickly

381 identify and extract the main plastome regions in a coordinated, standardized arrangement. This

382 new reference system not only will allow to define a global reorganization for the main plastome

383 regions but can also be employed to generate a chain of re-repositioning for all the remaining 
384 GenBank annotations in the sequence. This last condition applies to all plastomes in GenBank in

385 which the string starts randomly along the circular sequence. This allows to recast the previous

386 reference annotation into a new coordinate system for all the available annotations and print this 387 out in a GFF3 output file. In addition, the generation of reorganized and reoriented datasets, thus

388 already formatted for downstream analyses (e.g. alignment) will greatly improve the utility of 389 plastomes, either newly sequenced or mined from GenBank. Finally, the new coordinate system 390 can be used to implement exploratory methods for a more accurate and faster analysis of 391 phylogenetic comparative data, either using complete regions or concrete molecular markers in 392 case of using GenBank files. This, in turn, will allow us to advance the development of more 393 accurate hypotheses in the reconstruction of the evolutionary history of extant plant groups.

\section{Conclusions}

396 Curating draft plastomes and formatting them for downstream phylogenetic analyses is laborious,

397 time-consuming and error-prone. We developed ECUADOR for the robust extraction and mass 398 reorganization of plastome regions. The proposed algorithm is based on sliding windows and 399 dynamic suffix array approaches to track inverted repeat locations, followed by extraction and 400 repositioning of the main chloroplast regions. In addition, the user can generate datasets in which 401 all sequences are similarly oriented, allowing a direct inference of the homology through 402 sequence alignment. This facilitates post-processing analyses of extra-nuclear genomes from 403 NGS data, optimizing handling times and reducing error. We demonstrated its accuracy, 404 especially in handling poorly reconstructed plastomes, when repeats are interrupted by 405 misassembled positions (resulting in fragments poorly positioned throughout the sequence), 406 preventing recovery of IRs. This method significantly reduces handling time and complexity in 407 the analysis of large plastome datasets and allows for error free processing of high quantities of 408 data. Our study not only underscores the importance of developing new tools for detecting and 409 characterizing inverted repeated sequences, but also provides a new approach to systematically 410 identify complete regions within plastomes. ECUADOR will be maintained and regularly 411 improved to add new features, according to the emergence of new needs with the development of 412 innovative approaches in NGS. For example, future scheduled improvements include the 413 extraction of the homologous genes and non-coding regions (i.e. intergenic spacers, introns, and 414 ribosomal RNA) to generate locus-specific alignments, as currently widely used to avoid the 415 laborious steps involved in manual curation. We believe ECuADOR has the potential to be useful 
416 and widely applicable to the plant science community that handles large genomic datasets on a 417 daily basis, whether this is for genomics/phylogenomics, evolution, ecology or bioinformatics. 418

419 Acknowledgements

420 We kindly thank F. Areces-Berazain for providing useful comments to an earlier version of this 421 manuscript. 


\section{References}

423 1. McPherson H, Van der Merwe M, Delaney SK, Edwards MA, Henry RJ, McIntosh E, Rymer

424 PD, Milner ML, Siow J, \& Rossetto M. Capturing chloroplast variation for molecular ecology

425 studies: a simple next generation sequencing approach applied to a rainforest tree. BMC Ecol. 426 2013;13:8.

427 2. Scarcelli N, Mariac C, Couvreur TLP, Faye A, Richard D, Sabot F, Berthouly-Salazar C, \& 428 Vigouroux Y. Intra-individual polymorphism in chloroplasts from NGS data: Where does it come 429 from and how to handle it? Mol Ecol Resour. 2016;16(2):434-445.

430 3. Ruhfel BR, Gitzendanner MA, Soltis PS, Soltis DE, Burleigh JG. From algae to angiosperms-

431 inferring the phylogeny of green plants (Viridiplantae) from 360 plastid genomes. BMC Evol 432 Biol. 2014;14(1):23.

433 4. Twyford AD, Ness RW. Strategies for complete plastid genome sequencing. Mol Ecol Resour. 434 2017;17(5):858-68.

435 5. Zhang YJ, Ma PF, Li DZ. High-throughput sequencing of six bamboo chloroplast genomes: 436 phylogenetic implications for temperate woody bamboos (Poaceae: Bambusoideae). PLoS One. 437 2011;6(5):e20596. doi:10.1371/journal.pone.0020596.

438 6. Kress WJ. Plant DNA barcodes: applications today and in the future. JSE. 2017; 439 https://doi.org/10.1111/jse.12254.

440 7. Stull GW, Moore MJ, Mandala VS, Douglas NA, Kates HR, Qi X, Brockington SF, Soltis PS, 441 Soltis DE, \& Gitzendanner MA. A targeted enrichment strategy for massively parallel sequencing 442 of angiosperm plastid genomes. Appl Plant Sci. 2013;1(2):1200497.

443 8. de Abreu NL, Alves RJV, Cardoso SRS, Bertrand YJK, Sousa F, Hall CF, Pfeil BE, \& 444 Antonelli A. The use of chloroplast genome sequences to solve phylogenetic incongruences in 445 Polystachya Hook (Orchidaceae Juss). PeerJ. 2018;6:e4916.

446 9. Li H-T, Yi T-S, Gao L-M, Ma P-F, Zhang T, Yang J-B, Gitzendanner MA, Fritsch PW, Cai J, 447 Luo Y, Wang H, Van der Bank M, Zhang S-D, Wang Q-F, Wang J, Zhang Z-R, Fu C-N, Yang J, 448 Hollingsworth PM, Chase MW, Soltis DE, Soltis PS, Li D-Z. 2019. Origin of angiosperms and 449 the puzzle of the Jurassic gap. Nature Plants 5(5):461-470.

450 10. Palmer JD. Comparative organization of chloroplast genomes. Annual Review of Genetics. 451 1985;19(1):325-354.

452 11. Stadermann KB, Holtgräwe D, Weisshaar B. Chloroplast genome sequence of Arabidopsis 453 thaliana accession Landsberg erecta, assembled from single-molecule, real-time sequencing data. 454 Genome Announc. 2016;4(5):e00975-16. 
455 12. Shaw J, Lickey EB, Beck JT, Farmer SB, Liu W, Miller J, Siripun KC, Winder CT, Schilling 456 EE, \& Small RL. 2005. The tortoise and the hare II: relative utility of 21 noncoding chloroplast 457 DNA sequences for phylogenetic analysis. American Journal of Botany 92:142-166.

458 13. Graham SW, Reeves PA, Burns AC, Olmstead RG. Microstructural changes in noncoding 459 chloroplast DNA: interpretation, evolution, and utility of indels and inversions in basal 460 angiosperm phylogenetic inference. Int. J. Plant Sci. 2000;161(S6) Suppl 83-96.

461 14. Borsch T, Hilu KW, Quandt D, Wilde V, Neinhuis C, Barthlott W. Noncoding plastid trnT462 trnF sequences reveal a well resolved phylogeny of basal angiosperms. J Evol Biol. 463 2003;16(4):558-576

464 15. Gruenstaeudl M, Gerschler N, Borsch T. Bioinformatic workflows for generating complete 465 Plastid genome sequences-An example from Cabomba (Cabombaceae) in the context of the 466 phylogenomic analysis of the water-lily clade. Life. 2018;8(3):25

467 16. Castandet B, Hotto AM, Strickler SR, Stern DB. ChloroSeq, an optimized chloroplast RNA468 Seq Bioinformatic pipeline, reveals remodeling of the Organellar transcriptome under heat stress. 469 G3: Genes, Genomes, Genetics. 2016;6(9):2817-27.

470 17. Jin JJ, Yu WB, Yang JB, Song Y, Yi TS, Li DZ. GetOrganelle: a simple and fast pipeline for 471 de novo assembly of a complete circular chloroplast genome using genome skimming data. 472 BioRxiv. 2018;256479.

473 18. Bi G, Mao Y, Xing Q, Cao M. HomBlocks: a multiple-alignment construction pipeline for 474 organelle phylogenomics based on locally collinear block searching. Genomics. 2018;110(1):1847522.

476 19. Granados Mendoza C, Wanke S, Salomo K, Goetghebeur P, Samain MS. Application of the 477 phylogenetic informativeness method to chloroplast markers: A test case of closely related 478 species in tribe Hydrangeeae (Hydrangeaceae). Mol Phylogenet Evol. 2013;66:233-42.

479 20. Brázda V, Lýsek J, Bartas M, Fojta M. Complex Analyses of Short Inverted Repeats in All 480 Sequenced Chloroplast DNAs. Biomed Res. Int. 2018;2018.

481 21. Ripma LA, Simpson MG, Hasenstab-Lehman K. Geneious! simplified genome skimming 482 methods for phylogenetic systematic studies: A case study in oreocarya (boraginaceae). Appl 483 Plant Sci. 2014;2(12):1-12.

484 22. Katoh K, Standley DM. MAFFT multiple sequence alignment software version 7: 485 improvements in performance and usability. Mol Biol Evol. 2013;30(4):772-80. 
486 23. Guindon S, Dufayard JF, Lefort V, Anisimova M, Hordijk W, Gascuel O. New algorithms 487 and methods to estimate maximum-likelihood phylogenies: assessing the performance of PhyML 488 3.0. Syst Biol. 2010;59(3):307-321.

489 24. Wicke S, Müller KF, de Pamphilis CW, Quandt D, Wickett NJ, Zhang Y, Renner SS, 490 Schneeweiss GM. Mechanisms of functional and physical genome reduction in photosynthetic 491 and nonphotosynthetic parasitic plants of the broomrape family. Plant Cell. 2013;25(10):371149225.

493 25. Wicke S, Müller KF, dePamphilis CW, Quandt D, Bellot S, Schneeweiss GM. Mechanistic 494 model of evolutionary rate variation en route to a nonphotosynthetic lifestyle in plants. 495 Proceedings of the National Academy of Sciences. 2016;113(32):9045-9050.

496 26. Hunt M, Silva ND, Otto TD, Parkhill J, Keane JA, Harris SR. Circlator: automated 497 circularization of genome assemblies using long sequencing reads. Genome Biol. 2015;16(1). 498 doi:10.1186/s13059-015-0849-0.

499 27. Herbig A, Jager G, Battke F, Nieselt K. GenomeRing: alignment visualization based on 500 SuperGenome coordinates. Bioinformatics. 2012;28:i7-15. 10.1093/bioinformatics/bts217.

501 28. Maurer M, Molidor R, Sturn A, Hartler J, Hackl H, Stocker G, Prokesch A, Scheideler M, 502 Trajanoski Z. MARS: microarray analysis, retrieval, and storage system. BMC Bioinformatics 503 2005;6(1):101. 10.1186/1471-2105-6-101

504 29. McKain MR, Wilson M. Fast-Plast: rapid de novo assembly and finishing for whole 505 chloroplast genomes. 2017. https://github.com/mrmckain.

506 30. Chase MW, Christenhusz MJ, Fay MF, Byng JW, Judd WS, Soltis DE, Mabberley DJ, 507 Sennikov AN, Soltis PS, Stevens PF. An update of the Angiosperm Phylogeny Group 508 classification for the orders and families of flowering plants: APG IV. Botanical Journal of the 509 Linnean Society. 2016;181(1):1-20.

510 31. Gonçalves DJ, Simpson BB, Ortiz EM, Shimizu GH, Jansen RK. Incongruence between gene 511 trees and species trees and phylogenetic signal variation in plastid genes. Molecular 512 phylogenetics and evolution. 2019;138:219-232.

513 32. Walker, J.F., Walker-Hale, N., Vargas, O.M., Larson, D.A. and Stull, G.W., 2019.

514 Characterizing gene tree conflict in plastome-inferred phylogenies. PeerJ, 7, p.e7747. 
515 Figures, tables and additional files

516

517 Figure 1. Schematic representation of plastomes and effects of the artifactual linearisation during the assembly process. IRa and IRb: Inverted Repeats; LSC Large Single Copy region; SSC Small Single Copy region. a) Circular representation showing the potential cuts (numbered black arrows) during assembly (approximate positions); green arrow conventional start of the plastome sequence (resulting in the structure LSC-IRb-SSC-IRa); usual approximate sizes for each region are indicated. b) linear representations of a circular plastome, cut according to the black arrows in a); line numbers according to a). Note that IRs are split in three fragments in configurations 5 and 9.

Figure 2. Flow chart of the ECUADOR script.

Figure 3. Phylogenetic tree constructed with 161 cpDNAs, using fast likelihood-based method (aLRT SH-like) as implemented in PhyML [23]. Numbers on nodes indicate probability values. Families highlighted in red show an inconsistency found in the placement of Ranunculaceae (Ranunculus macranthus), which groups together with Piperaceae, Dioscoreaceae and Chloranthaceae.

Figure 4. Accuracy of $E C U A D O R$ in retrieving the correct IR locations in plastomes of decreasing quality. Vertical axis percentage of simulations where correct (grey) or incorrect (yellow) IR locations were retrieved. Horizontal axis: Percentage of mismatching positions introduced in the IR sequences of the Arabidopsis thaliana reference plastome sequence (NC_000932) for 1000 simulations. Values in the lower part shows the total assigned variation in base pairs for each set respectively. Red values below the bars show the error average in base pairs for the positioning of the uncertain annotation.

\section{Supplementary information}


542 Supplementary table S1. ECuADOR results for dataset1. Dataset1 containing 53 major

543 angiosperm groups used in our study obtained from [3] as well as the analysis of their 161

544 evaluated species. Positions and length of the large single copy (LSC), inverted repeat-B

545 (IRb), short single copy (SSC) and inverted repeat-A (IRa) are shown. In addition, these

546 results were compared with the "Find repeats" function in Geneious R9 v.9.0.5 to confirm

547 successful matches, validating the performance and accuracy.

548 Supplementary Table S2. Analysis results of 1000 simulations with dataset2 and the

549 Arabidopsis thaliana reference plastome sequence (NC_000932). (PDF)

550 Supplementary file S1. Dataset3, corresponding to 4446 angiosperm chloroplasts, was

551 used in our evaluation. These chloroplast genomes were downloaded from GenBank, using

552 the database INSDC (accessed on 2019/06/20 - dataset3: mass data evaluation). (TXT)

553 Supplementary file S2. Dataset3, corresponding to 95 angiosperm chloroplasts where the

554 main regions were not detected. (TXT)

555

556 


\section{Figure 1}

Schematic representation of plastomes and effects of the artifactual linearisation during the assembly process

Schematic representation of plastomes and effects of the artifactual linearisation during the assembly process. IRa and IRb: Inverted Repeats; LSC Large Single Copy region; SSC Small Single Copy region. a) Circular representation showing the potential cuts (numbered black arrows) during assembly (approximate positions); green arrow conventional start of the plastome sequence (resulting in the structure LSC-IRb-SSC-IRa); usual approximate sizes for each region are indicated. b) linear representations of a circular plastome, cut according to the black arrows in a); line numbers according to a). Note that IRs are split in three fragments in configurations 5 and 9.
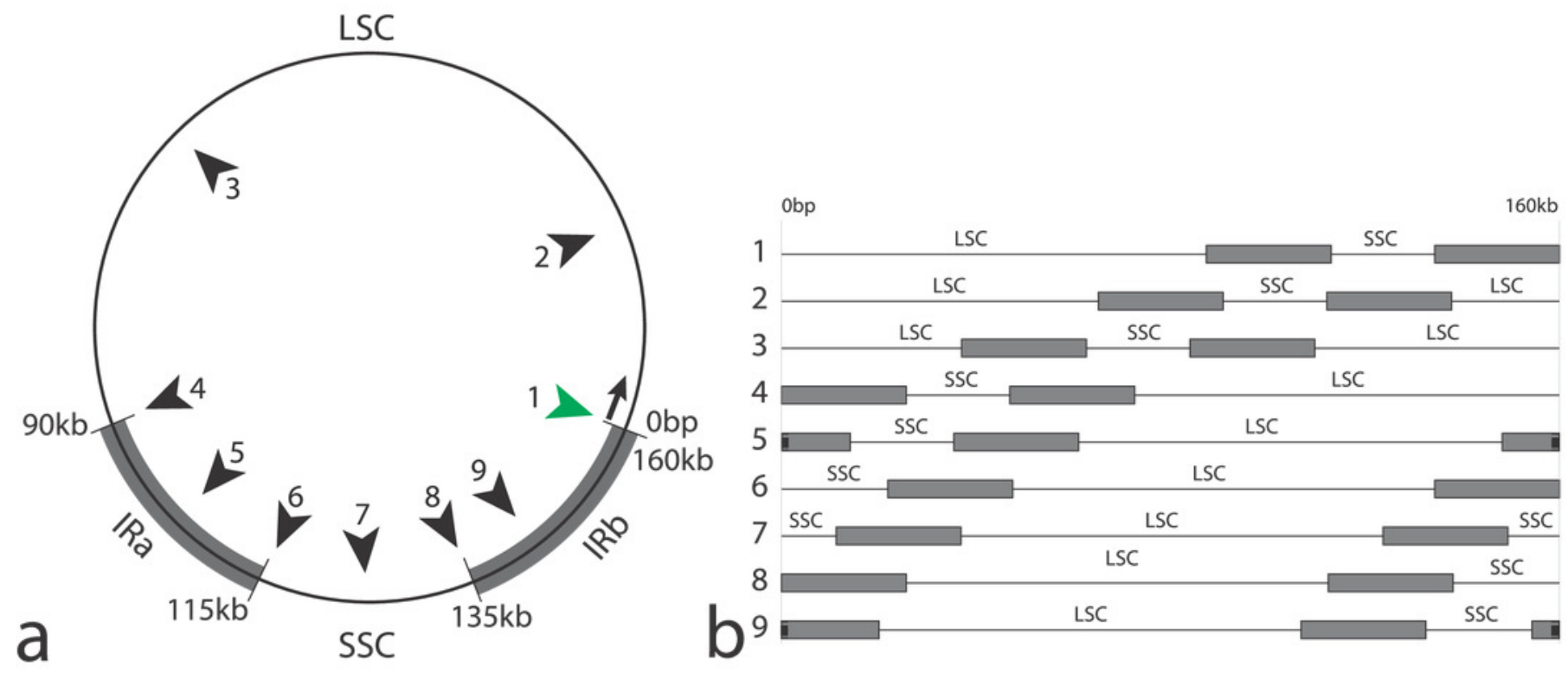
Figure 2

Flow chart of the ECuADOR script.

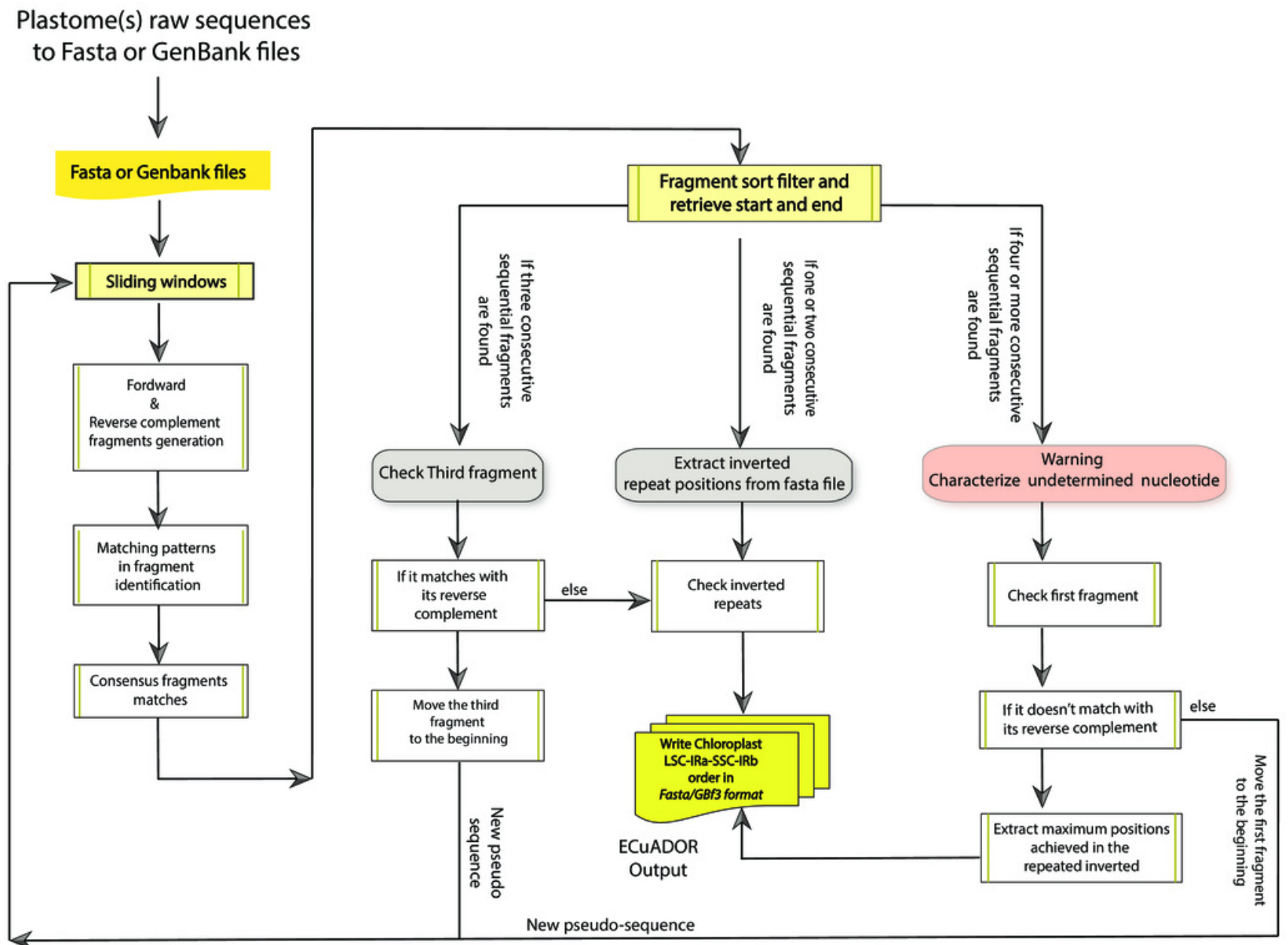




\section{Figure 3}

Phylogenetic tree constructed with 161 cpDNAs, using fast likelihood-based method (aLRT SH-like) as implemented in PhyML [23].

Phylogenetic tree constructed with 161 cpDNAs, using fast likelihood-based method (aLRT SH-like) as implemented in PhyML [23]. Numbers on nodes indicate probability values.

Families highlighted in red show an inconsistency found in the placement of Ranunculaceae (Ranunculus macranthus), which groups together with Piperaceae, Dioscoreaceae and Chloranthaceae. 


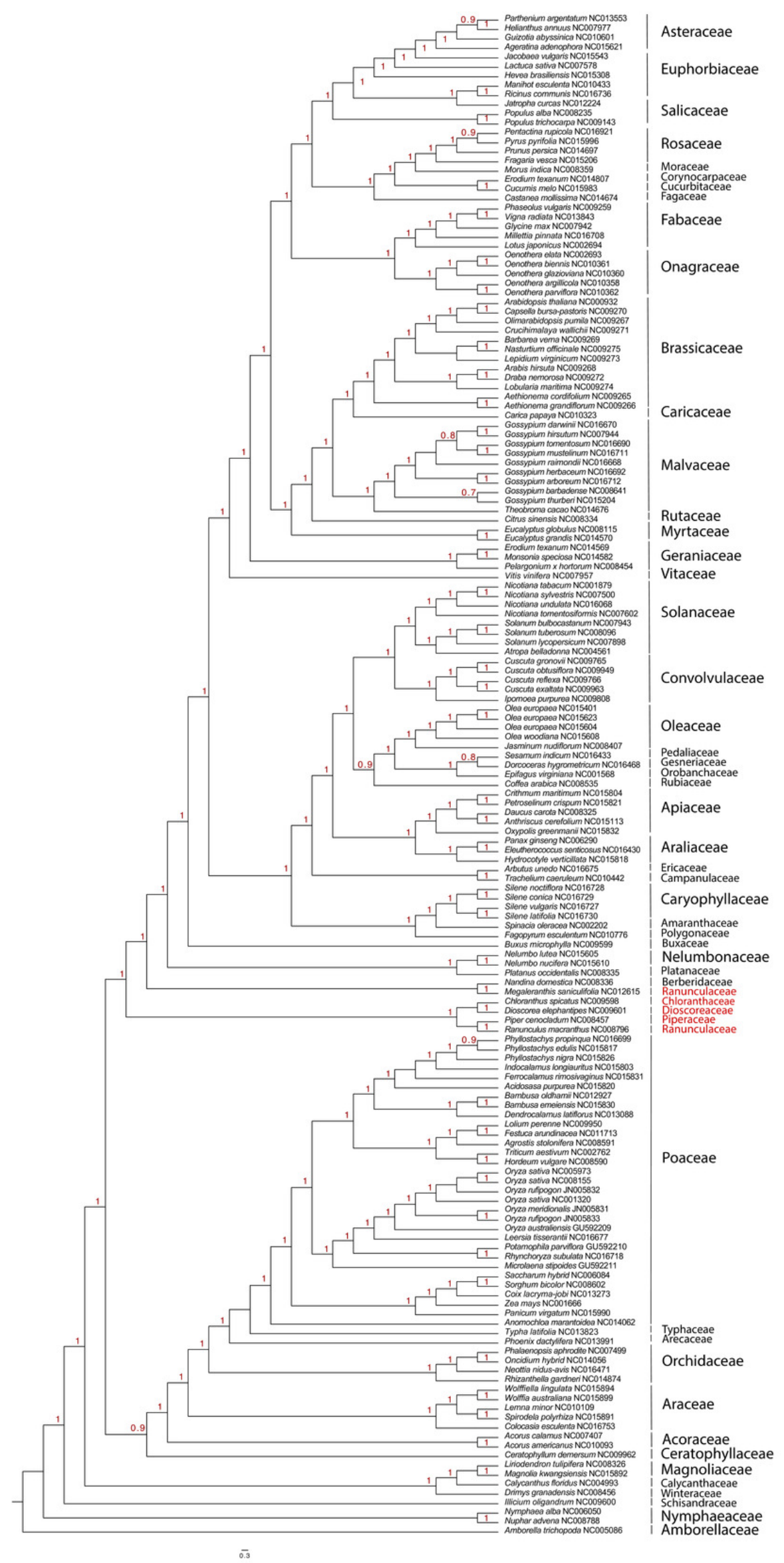




\section{Figure 4}

Accuracy of ECUADOR in retrieving the correct IR locations in plastomes of decreasing quality

Vertical axis percentage of simulations where correct (grey) or incorrect (yellow) IR locations were retrieved. Horizontal axis: Percentage of mismatching positions introduced in the IR sequences of the Arabidopsis thaliana reference plastome sequence (NC_000932) for 1000 simulations. Values in the lower part shows the total assigned variation in base pairs for each set respectively. Red values below the bars show the error average in base pairs for the positioning of the uncertain annotation.

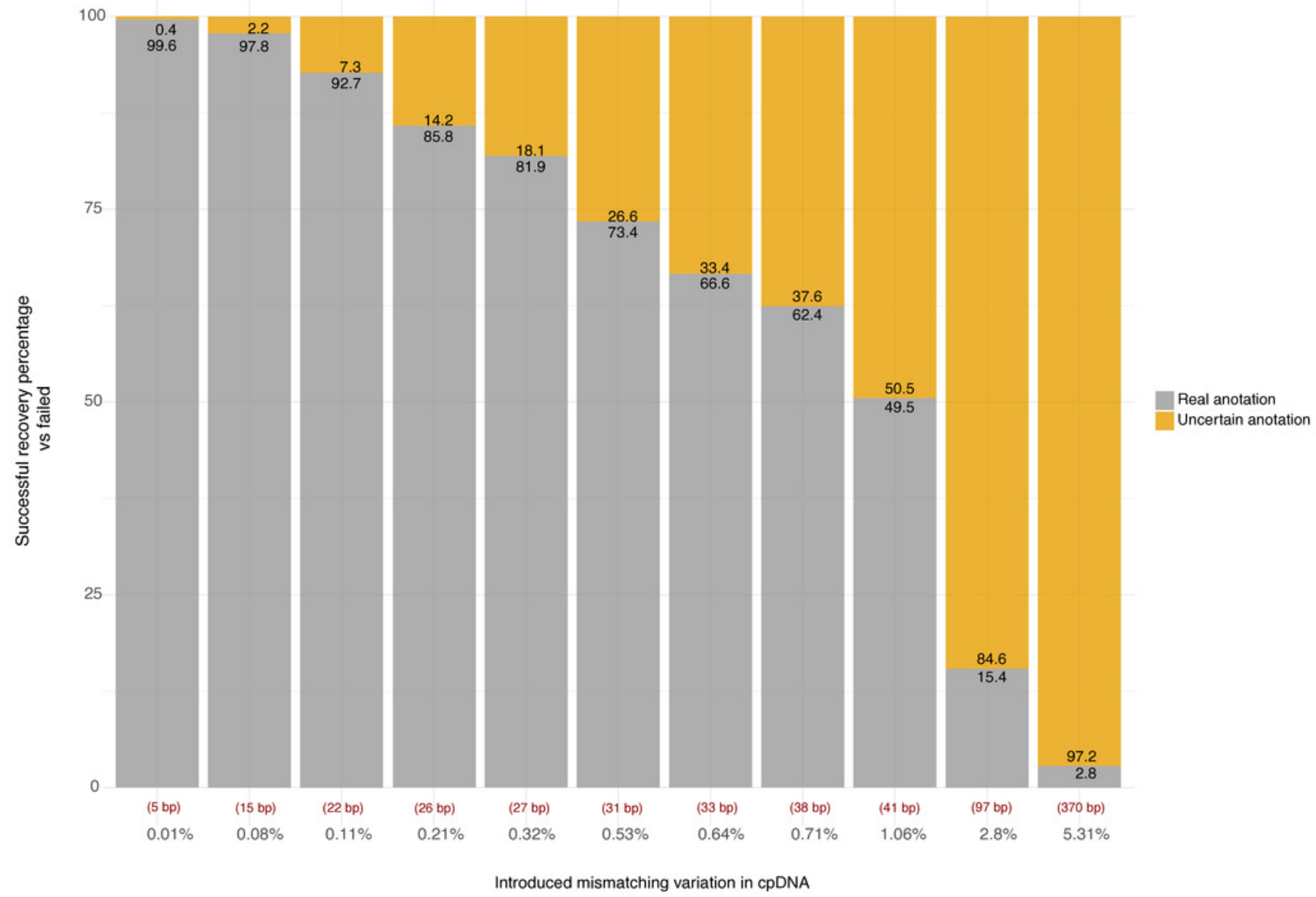

